\title{
(1) PRDDUCுनि口 DNLINE

\section{IMPACTO FINANCEIRO DOS ERROS NA PREVISÃO EMPRESARIAL: UM ESTUDO COMPARATIVO ENTRE MODELOS LINEARES E REDES NEURAIS}

\section{FINANCIAL IMPACT OF ERRORS IN BUSINESS FORECASTING: A COMPARATIVE STUDY OF LINEAR MODELS AND NEURAL NETWORKS}

\author{
Claudimar Pereira da Veiga* E-mail: Claudimar.veiga@gmail.com \\ Cassia Rita Pereira da Veiga* E-mail: cassia.veig@gmail.com \\ Guilherme Ernani Vieira* E-mail: gui.vieira@pucpr.br \\ Ubiratã Tortato* E-mail: ubirata.tortato@pucpr.br \\ *Pontifícia Universidade Católica do Paraná - PUCPR, Curitiba, PR
}

\begin{abstract}
Resumo: A importância da previsão de demanda como uma ferramenta gerencial é um assunto bem documentado. Entretanto, é difícil mensurar os custos gerados por erros de previsão, assim como encontrar no mercado um modelo que assimile adequadamente as particularidades do funcionamento de cada empresa. No geral, quando os modelos lineares falham no processo de previsão, modelos não lineares mais complexos são considerados. Embora alguns estudos comparativos entre os modelos tradicionais e redes neurais tenham sido conduzidos na literatura, as conclusões são geralmente contraditórias. Neste sentido, o objetivo deste trabalho foi comparar a acurácia de métodos de previsão de demanda lineares e redes neurais em relação ao atual método utilizado pela empresa em estudo. Os resultados desta análise também serviram como subsídio para avaliar a influência dos erros de previsão de demanda no desempenho financeiro da empresa. $O$ estudo foi conduzido em cinco grupos de produtos, com dados históricos de demanda abrangendo o período de 2004 a 2008. De um modo geral, pode-se afirmar que todos os modelos estudados apresentaram uma previsão boa (melhor que o atual método usado pela empresa), com erro absoluto médio percentual (MAPE) em torno de 10\%. O impacto financeiro total referente aos erros de previsão para a empresa foi de aproximadamente $6,05 \%$ sobre o faturamento anual.
\end{abstract}

Palavras-Chaves: Previsão de demanda. Métodos lineares. Redes neurais. Acuracidade. Desempenho financeiro.

Abstract: The importance of demand forecasting as a management tool is a well documented issue. However, it is difficult to measure costs generated by forecasting errors and to find a model that assimilate the detailed operation of each company adequately. In general, when linear models fail in the forecasting process, more complex nonlinear models are considered. Although some studies comparing traditional models and neural networks have been conducted in the literature, the conclusions are usually contradictory. In this sense, the objective was to compare the accuracy of linear methods and neural networks with the current method used by the company. The results of this analysis also served as input to evaluate influence of errors in demand forecasting on the financial performance of the company. The study was based on historical data from five groups of food products, from 2004 to 2008. In general, one can affirm that all models tested presented good results (much better than the current forecasting method used), with mean absolute percent error (MAPE) around $10 \%$. The total financial impact for the company was $6,05 \%$ on annual sales. 
Palavras-Chaves: Demand forecasting. Linear models. Neural networks. Accuracy. financial performance.

\section{INTRODUÇÃO}

Em um mundo competitivo e globalizado, a previsão de demanda assume um papel primordial para o planejamento e operações dos negócios varejistas, seja no nível macro ou microeconômico. No nível organizacional, as previsões de vendas são necessárias para os inputs essenciais nas tomadas de decisão e envolvem os departamentos de marketing, vendas, produção, compras, logística e finanças.

Não basta, entretanto, ter um sistema de previsão de demanda na organização. É a qualidade da informação obtida por este sistema que capacita a organização a obter melhor planejamento de suas operações. Para KUO e XUE (1999), obter uma previsão de demanda precisa é o ponto crítico da qualidade do processo decisório. A previsão tenta calcular e predizer uma circunstância futura providenciando a melhor avaliação da informação comercial disponível.

A importância da acuracidade da previsão de demanda é um assunto bem documentado. Estudos recentes demonstram que uma previsão de demanda de alta acurácia assume uma função crítica para a lucratividade varejista ( $\mathrm{CHU}$ e ZHANG, 2003) e exerce uma influência direta no nível de serviço oferecido ao consumidor, no nível de estoque de segurança e no custo total da cadeia de suprimentos. Se uma previsão é mais acurada significa que a produção pode antecipar melhor a demanda do cliente (MEIJDEN, NUNEN e RAMONDT, 1994). Ao contrário, uma previsão inadequada pode comprometer os resultados da cadeia de suprimentos e gerar três situações: stockouts (não atendimento de demanda), backlogs (demanda atendida com atraso) e/ou excesso de estoque. Estas situações, além de aumentar os custos do produto, comprometem o fluxo de caixa e a rentabilidade da organização.

Os negócios varejistas frequentemente exibem forte variação sazonal. Este é um dos grandes desafios da indústria de alimentos: realizar o planejamento da produção em acompanhamento às necessidades de demanda. Historicamente, prever dados sazonais e com tendência representa um dos principais esforços de pesquisa em gestão de estoque e muitos métodos teóricos 
e heurísticos têm sido desenvolvidos nas últimas décadas. As abordagens quantitativas tradicionais incluem métodos heurísticos tais como decomposição de séries temporais, suavização exponencial e médias móveis auto-regressivas integradas (ARIMA). A principal limitação destes métodos tradicionais é que eles são essencialmente lineares. No geral, nos métodos lineares, os usuários não precisam conhecer a relação complexa entre os dados. Como vantagem prática, estes modelos são de fácil interpretação e implementação (CHU e ZHANG, 2003, CHEN, 2011).

Geralmente quando os modelos lineares falham no processo de previsão, modelos não lineares mais complexos devem ser considerados (CHU e ZHANG, 2003, CHEN, 2011). Deste modo, muitos estudos para a previsão de demanda utilizam métodos não lineares como redes neurais (RN) e regressão de vetor de suporte (LEVIS e PAPAGEORGIOU, 2005). Estes métodos são projetados para captar padrões não lineares da série temporal, por isso apresentam desempenho superior para modelar o comportamento econômico e para tomadas de decisão subjacentes mais complexas.

É difícil mensurar os custos gerados pelo erro de previsão, assim como encontrar no mercado um modelo que assimile adequadamente as particularidades do funcionamento de cada empresa. Portanto, diante de diferentes possibilidades de métodos de previsão, torna-se necessário identificar por índices de desempenho qual a metodologia mais adequada para cada caso. Embora numerosos estudos comparativos entre os modelos lineares e redes neurais tenham sido conduzidos na literatura, os achados não demonstram consenso, pelo contrário, as conclusões são contraditórias (CHU e ZHANG, 2003). Além disso, muitos trabalhos comparam diversos modelos de previsão, mas não pesquisam o uso das informações obtidas no processo de tomada de decisão ou no impacto na cadeia de suprimentos como um todo.

Neste contexto, o objetivo do presente trabalho é comparar a acurácia de métodos lineares de previsão de demanda (suavização exponencial simples, modelo de Holt, modelo de Winter e ARIMA) e de um método não linear (redes neurais) em relação à atual metodologia utilizada pela empresa em estudo. Os resultados desta análise servirão como subsídio para avaliar a influência dos erros de previsão de demanda no desempenho financeiro da empresa, utilizando o fill rate como indicador 
do nível de serviço oferecido ao consumidor, assim como do custo de oportunidade em resposta à demanda. $O$ estudo será conduzido em cinco grupos de produtos do portfólio de uma empresa varejista, com dados históricos de demanda abrangendo o período de 2004 a 2008.

\section{FUNDAMENTOS SOBRE OS MÉTODOS DE PREVISÃO CONSIDERADOS}

A previsão da demanda é um assunto extremamente difundido e aceito como parte integral dos planos de negócios e tomadas de decisão (MAKRIDAKIS, WHEELWRIGHT e HYNDMAN, 1998, NEUMANN, 2010, ROMUALDO, BAPTISTA e VIEIRA, 2010, ALI, BOYLAN e SYNTETOS, 2011). De uma perspectiva histórica, os métodos de suavização exponencial e de decomposição foram os primeiros modelos de previsão a serem desenvolvidos, o que ocorreu na década de 1950 (SOUZA, SAMOHYL e MIRANDA, 2008). Durante a década de 1960, a introdução dos computadores tornou mais disponível e menos onerosa a aplicação de metodologias mais sofisticadas, tais como as médias móveis auto-regressivas integradas (ARIMA) desenvolvidas por Box e Jenkins em 1969 (BOX, JENKINS e REINESL, 1994). Na sequência, durante as décadas de 1970 e 1980, novas abordagens foram introduzidas como técnicas de previsão incluindo métodos econométricos e Bayesianos (MAKRIDAKIS, WHEELWRIGHT e HYNDMAN, 1998). Entretanto, a consolidação e a melhoria dos modelos de previsão ocorreram somente na década de 1990 com a emergência das redes neurais artificiais, uma metodologia em que o computador desempenha funções habitualmente realizadas pela inteligência humana tais como razão, aprendizagem e melhorias próprias (DELURGIO, 1998, ZHANG, 2003, HAYKIN, 2001).

Apesar da evolução que ocorreu na metodologia empregada para a previsão, é importante ressaltar que estudos empíricos demonstram que o aumento da complexidade do modelo nem sempre é acompanhado de aumento na acurácia preditiva (TERASVIRTA, DIJK e MEDEIROS, 2005). "A busca da redução das discrepâncias sem se afastar da simplicidade e praticidade de determinadas técnicas de previsão é uma combinação sempre bem vinda em ambientes dinâmicos como os empresariais" (SOUZA, SAMOHYL e MIRANDA, 2008: 117). Além disso, estudos recentes demonstram que formulações simples na nova geração de 
ferramentas de previsão compartilham dos mesmos benefícios das redes neurais artificiais (LEVIS e PAPAGEORGIOU, 2005). Maiores informações sobre os métodos de previsão de demanda podem ser encontradas nos Quadros 1, 2 e 3.

Quadro 1 - Descrição dos modelos quantitativos lineares que não consideram tendência e/ou sazonalidade contidas na série temporal

\begin{tabular}{|c|c|c|}
\hline Modelos & Fórmula & Aplicação \\
\hline $\begin{array}{l}\text { Médias Móveis } \\
\text { Simples (MMS) }\end{array}$ & $\begin{array}{l}M_{\mathrm{t}}=\frac{Z_{\mathrm{s}}+Z_{\mathrm{s}-1}+\cdots+Z_{\mathrm{s}-\mathrm{r}+1}}{r} \\
M_{\mathrm{t}}=\text { estimativa do nível } \\
\mathrm{Z}_{\mathrm{t}}=\text { dado de cada período } \\
\mathrm{r}=\text { media dos períodos } \\
\text { (Makridakis, Wheelwright e Hyndman, 1998) } \\
\text { (Faria, Albuquerque, Alfonso e et al, 2008) } \\
\text { (DeLúrgio, 1998) }\end{array}$ & $\begin{array}{l}\text { Para demanda } \\
\text { sem tendência ou } \\
\text { sazonalidade. } \\
\text { Método simples, } \\
\text { de fácil } \\
\text { implantação. }\end{array}$ \\
\hline $\begin{array}{c}\text { Suavização } \\
\text { Exponencial } \\
\text { Simples }\end{array}$ & $\begin{array}{l}\overline{\mathrm{Z}}_{\mathrm{t}}=\boldsymbol{\alpha} \overline{\mathrm{Z}}_{\mathrm{t}}+\boldsymbol{\alpha}(\mathbf{1}-\boldsymbol{\alpha}) \overline{\mathrm{Z}}_{\mathrm{t}-1}+\boldsymbol{\alpha}(\mathbf{1}-\boldsymbol{\alpha})^{2} \overline{\mathrm{Z}}_{\mathrm{t}-2}+\ldots \\
\overline{\mathrm{Z}}_{\mathrm{t}}=\text { valor exponencial suavizado } \\
\alpha=\text { constante de suavização } \\
\text { (Souto, Baldeon e Russo, 2006) } \\
\text { (Martinez e Zamprogno, 2003) } \\
\text { (Taylor, 2007) }\end{array}$ & $\begin{array}{l}\text { Para demanda } \\
\text { sem tendência ou } \\
\text { sazonalidade. } \\
\text { Utiliza ajuste no } \\
\text { erro da última } \\
\text { previsão. }\end{array}$ \\
\hline
\end{tabular}

Fonte: VEIGA, VEIGA e DUCLÓS, 2010

Atualmente, existem pelo menos 70 técnicas diferentes entre métodos lineares e não lineares para a previsão quantitativa de demanda (KERKANEN, KORPELA e HUISKONEN, 2009). Apesar disso, o conceito básico da grande maioria é o mesmo: os padrões de comportamento do passado continuarão no futuro, ou seja, assume-se que a demanda de um período de tempo passado será equivalente à demanda de um período correspondente no futuro (MORETTIN e TOLOI, 1987, DELURGIO, 1998, MAKRIDAKIS, WHEELWRIGHT e HYNDMAN, 1998, CHOPRA e MEINDL, 2003). Estas técnicas utilizam diferentes modelos matemáticos e exigem diferentes níveis de esforço e investimento. Utilizar técnicas complexas nem sempre é vantajoso, uma vez que o custo de se utilizar um modelo complexo pode facilmente superar o ganho marginal da redução no erro de previsão. Atingir a relação ótima entre custo e benefício exige entender as premissas, vantagens, desvantagens, aplicações e custos das principais abordagens de previsão de demanda (NEUMANN, 2010). 
Quadro 2 - Descrição dos modelos quantitativos lineares que consideram tendência e/ou sazonalidade contidas na série temporal

\begin{tabular}{|c|c|c|}
\hline Modelos & Fórmula & Aplicação \\
\hline $\begin{array}{l}\text { Modelo de } \\
\text { Holt }\end{array}$ & $\begin{array}{l}L_{t+1}=\alpha D_{t+1}+(1-\alpha)\left(L_{t}+T_{t}\right) \\
T_{t+1}=\beta\left(L_{t+1}-L_{t}\right)+(1-\beta) T_{t} \\
L_{t}=\text { estimativa do nível do período } t \\
L_{t+1}=\text { estimativa do nível do período } t+1 \\
T_{t}=\text { estimativa de tendência do período } t \\
T_{t+1}=\text { estimativa de tendência do período } t+1 \\
D_{t+1}=\text { demanda real observada no período } t+1 \\
\alpha=\text { constante de suavização para o nível } \\
B=\text { constante de suavização para a tendência } \\
\text { (Delurgio, 1998) } \\
\text { (Holt, 2004) } \\
\text { (Martinez e Zamprogno, 2003) }\end{array}$ & $\begin{array}{l}\text { Para demanda } \\
\text { que apresenta } \\
\text { tendência. } \\
\text { Requer a } \\
\text { utilização de } \\
\text { pacotes } \\
\text { computacionais }\end{array}$ \\
\hline $\begin{array}{l}\text { Modelo de } \\
\text { Winter }\end{array}$ & $\begin{array}{l}\mathrm{L}_{t+1}=\alpha\left(D_{t+1} S_{t+1}\right)+(1-\alpha)\left(L_{t}+T_{t}\right) \\
T_{t+1}=\beta\left(L_{t+1}-L_{t}\right)+(1-\beta) T_{t} \\
S_{t+p+1}=Y\left(D_{t+1} L_{t+1}\right)+(1-Y) S_{t+1} \\
L_{t}=\text { estimativa do nível do período } t \\
L_{t+1}=\text { estimativa do nível do período } t+1 \\
T_{t}=\text { estimativa de tendência do período } t \\
T_{t+1}=\text { estimativa de tendência do período } t+1 \\
S_{t+1}=\text { estimativa do fator de sazonalidade } t+1 \\
S_{t+p+1}=\text { estimativa do fator de sazonalidade } \\
t+p+1 \\
D_{t+1}=\text { demanda real observada no período } t+1 \\
\alpha=\text { constante de suavização para o nível } \\
\beta=\text { constante de suavização para a tendência } \\
Y=\text { constante de suavização para a } \\
\text { sazonalidade } \\
\text { (Delurgio, 1998) } \\
\text { (Segura, 2001) }\end{array}$ & $\begin{array}{c}\text { Para demanda que } \\
\text { apresenta } \\
\text { tendência e/ou } \\
\text { sazonalidade. } \\
\text { Facilidade de } \\
\text { interpretação dos } \\
\text { índices de } \\
\text { sazonalidade e } \\
\text { entendimento } \\
\text { gerencial. Pode se } \\
\text { adequar a } \\
\text { eficientes } \\
\text { algoritmos } \\
\text { computacionais. }\end{array}$ \\
\hline $\begin{array}{l}\text { Modelo } \\
\text { ARIMA }\end{array}$ & $\begin{array}{l}\phi(B)\left[(1-B)^{d} y_{t}-\mu\right]=\theta(B) u_{t} \\
\phi=\text { parâmetros autoregressivos } \\
\theta=\text { parâmetro médias móveis } \\
\phi(B)=\text { polinômio de AR } \\
\theta(B)=\text { polinômios MA } \\
\text { (Box, Jenkins e Reinesl, 1994) } \\
\text { (Zang, 2003) }\end{array}$ & $\begin{array}{l}\text { Para séries } \\
\text { temporais que } \\
\text { apresentam auto- } \\
\text { correlação. Difícil } \\
\text { operacionalização. } \\
\text { Requer programas } \\
\text { computacionais. }\end{array}$ \\
\hline
\end{tabular}

Fonte: Veiga, Veiga e Duclós (2010)

A utilização de séries temporais permanece como a área de estudo mais importante em previsão de demanda. Nesta, as observações do comportamento passado de uma variável são coletadas e analisadas por modelos matemáticos para descrever as relações subjacentes. Este modelo é, então, utilizado para extrapolar a série temporal para o futuro (ZHANG, 2003, SONG e LI, 2008, CHEN, 2011). Esta abordagem é particularmente usual quando pouco 
conhecimento está disponível sobre o processo de geração dos dados ou quando não há um modelo ideal bem definido para a previsão futura.

Para estudar a previsão de demanda, os modelos de séries temporais podem ser classificados em duas categorias: métodos lineares e métodos nãolineares (CHU e ZHANG, 2003, CHEN, 2011). Os métodos lineares mais convencionais são média móvel simples (MMS), média móvel ponderada (MMP), suavização exponencial simples (SES), modelo de Holt (MH), modelo de Winter (MW) e médias móveis auto-regressivas integradas (ARIMA). No geral, nos métodos lineares, os usuários não precisam conhecer a relação complexa entre os dados. Como vantagem prática, estes modelos são de fácil interpretação e implementação. Entre os métodos lineares, o modelo ARIMA representa o método de previsão com resultados mais satisfatórios nas diversas aplicações empíricas a que foi submetido (CHU e ZHANG, 2003, SONG e LI, 2008, CHEN, 2011).

Geralmente quando não se obtém resultados satisfatórios com os modelos lineares no processo de previsão, modelos não lineares mais complexos devem ser considerados. Estes métodos são projetados para captar padrões não lineares da série temporal por isso apresentam bom desempenho para modelar o comportamento econômico e nas tomadas de decisão subjacentes.

O modelo não linear mais comumente utilizado é a rede neural artificial $(\mathrm{RN})$. A flexibilidade na condução dos dados tem feito da RN uma ferramenta atrativa já que com arquitetura apropriada, este método pode aproximar qualquer tipo de função com qualquer acurácia desejada (ZHANG, 2003, CHU e ZHANG, 2003). Sua grande desvantagem é a definição automática da estrutura da rede, que exige um grande número de testes e muita experiência por parte do projetista. Nesta situação, uma alternativa seria o SVR (Support Vector Regression), uma técnica de regressão não-linear que facilita a automatização e o trabalho em um espaço de alta dimensionalidade (LEVIS e PAPAGEORGIOU, 2005, CHEN, 2011, WANG, LI, NIU, et al, 2012). Dentre outros modelos não lineares pode-se citar o Takagi-Sugeno fuzzy system e Wavelet (KATATEPE, ALCI, 2005, ROMUALDO, BAPTISTA e VIEIRA, 2010) e SVM (Support Vector Machines) (TURRADO GARCIA, VILLALBA, PORTELA, 2009) novas técnicas capazes de lidar com grandes incertezas. 
O Instituto Internacional de Previsões foi estabelecido há aproximadamente 30 anos e tem a função de avaliar o progresso de previsões realizadas em séries temporais. Dentro deste período, vários métodos quantitativos de previsão de demanda foram aplicados e comparados em situações diversas, em produtos e mercados específicos. Embora diversos estudos comparativos tenham sido descritos na literatura, as conclusões não sugerem quais condições tornam um método melhor do que outro. Por isso, situações de complexidade, sazonalidade e perecibilidade, como ocorrem no mercado de alimentos, ainda exigem estudos investigativos sobre o método de previsão mais adequado para cada condição de estudo.

Quadro 3 - Descrição do método não-linear Redes Neurais feedforward e o algoritmo de LevenbergMarquadt

\begin{tabular}{|c|c|c|}
\hline Modelos & Fórmula & Aplicação \\
\hline Redes Neurais & $\begin{array}{l}y_{i}=f_{i}\left(\sum_{j=1}^{n} w_{i} x_{j}-\theta_{i}\right) \\
y_{i}=\text { saída do nó } i \\
x_{j}=j \text {-ésima entrada para o nó } \\
w_{i, j}=\text { peso da conexão entre os nós } \\
i \text { e } j \\
\theta_{i}=\text { bias do nó. } \\
f_{i}=\text { função não-linear } \\
\text { (Martinez e Zamprogno, 2003) } \\
\text { (Calôba, Calôba e Saliby, 2002) } \\
\text { (Zhang, 2003; Haykin, 2001) } \\
\text { (Koskela, 2003) }\end{array}$ & $\begin{array}{l}\text { As redes neurais } \\
\text { conseguem } \\
\text { eficiência destacada } \\
\text { quando tratam de } \\
\text { não linearidades. } \\
\text { A rede feedforward é } \\
\text { considerada um } \\
\text { modelo semi- } \\
\text { paramétrico em que } \\
\text { o número efetivo de } \\
\text { parâmetros } \\
\text { determina a } \\
\text { complexidade do } \\
\text { modelo. }\end{array}$ \\
\hline $\begin{array}{l}\text { Algoritmo de } \\
\text { Levenberg- } \\
\text { Marquardt }\end{array}$ & $\begin{array}{l}\qquad \Delta w=[H+\lambda]^{-1} g \\
\qquad w_{z+1}=w_{z}-\left(H+\lambda \text { disg }[H]^{-1} \Delta f\left(w_{t}\right)\right. \\
I=\text { matriz identidade de mesma dimensão que } H \\
\lambda=\text { regularização ou carregamento } \\
\text { (parâmetro que força a soma da matriz }(H+\lambda I) \text { a } \\
\text { um valor definitivamente positivo e seguramente } \\
\text { bem condicionado pelo computador) } \\
\text { (Levemberg, 1944) }\end{array}$ & $\begin{array}{l}\text { Eficiente para } \\
\text { solucionar o } \\
\text { problema de } \\
\text { minimização de } \\
\text { quadrados para } \\
\text { funções não- } \\
\text { lineares. }\end{array}$ \\
\hline
\end{tabular}

Fonte: Martinez e Zamprogno (2003); Calôba, Calôba e Saliby (2002); Zangh (2003), Haykin, (2001); Koskela (2003); Levemberg (1944)

Os trabalhos publicados referentes à comparação de modelos lineares e nãolineares geralmente utilizam os modelos ARIMA e Redes Neurais (ZHANG, 2003, CHU e ZHANG, 2003, HERAVI, OSBORN e BIRCHENHALL, 2004, GUTIERREZ- 
ESTRADA, PEDRO-SANZ, LOPEZ-LUQUE, et al, 2004, PAO, 2004, CHELANI e DEVOTTA, 2006, CO e BOOSARAWONGSE, 2007, OLIVEIRA, BERGMANN e FÁVERO, 2007, SANTOS, COSTA JR e COELHO, 2007, ZOU, XIA, YANG, et al, 2007, COELHO, SANTOS e COSTA JR, 2008, KOUTROUMANIDIS, IOANNOU e ARABATZIS, 2009, VEIGA, 2009). Esta comparação entre modelos lineares e nãolineares já foi avaliada para diversos produtos como previsão de energia (PAO, 2006), qualidade ambiental (GUTIERREZ-ESTRADA, PEDRO-SANZ, LOPEZLUQUE, et al, 2004, CHELANI e DEVOTTA, 2006), taxa de câmbio (HANN e STEURER, 1996, SANTOS, COSTA JR e COELHO, 2007, COELHO, SANTOS e COSTA JR, 2008), séries temporais macro-econômicas (SWANSON e WHITE, 1997, TERUI e DIJK, 2002, TERASVIRTA, DIJK e MEDEIROS, 2005), previsão de demanda de turismo (CHEN, 2011), vendas varejistas (CHU e ZHANG, 2003), preço no varejo (ZOU, XIA, YANG, et al, 2007, KOUTROUMANIDIS, IOANNOU e ARABATZIS, 2009), produção industrial (HERAVI, OSBORN e BIRCHENHALL, 2004), exportação (CO e BOOSARAWONGSE, 2007), retorno de ações (OLIVEIRA, BERGMANN e FÁVERO, 2007, SANTOS FILHO, SILVA, VEIGA, et al, 2011), entre outros.

No geral, a maioria dos artigos que comparam métodos lineares e RN demonstra que não há muito ganho em se utilizar um método de RN ao invés de um simples modelo linear autoregressivo (TERASVIRTA, DIJK e MEDEIROS, 2005, BARBOSA, WANKE, 2010), ou seja, os modelos parcimoniosos tendem a oferecer previsões mais precisas. Apesar disso, os diversos estudos comparativos demonstram que os resultados dependem de condições específicas da análise relacionadas aos produtos e mercados específicos.

Para a indústria de alimentos, em especial, poucos estudos que comparam métodos lineares e não-lineares foram publicados até o momento. Pode-se citar, por exemplo, a previsão de demanda de cerveja (CALÔBA, CALÔBA e SALIBY, 2002), da exportação de arroz (CO e BOOSARAWONGSE, 2007), de bebidas carbonatadas (SALGADO JÚNIOR, SEGATTO, CALIA, et al, 2010), do preço do grão (ZOU, XIA, YANG, et al, 2007) e do retorno de ações em empresas de alimentos (OLIVEIRA, BERGMANN e FÁVERO, 2007). Estes trabalhos científicos demonstram que o tema tem sido objeto de pesquisa apenas nos últimos anos, por 
este motivo ainda não foi possível explorar toda a complexidade que este segmento industrial apresenta.

A pesquisa de previsão tem comumente assumido que a acurácia é o critério primário na seleção do modelo de previsão ideal (CHU e ZHANG, 2003, ZHANG, 2003, TERASVIRTA, DIJK e MEDEIROS, 2005, PAO, 2006, COELHO, SANTOS e COSTA JR, 2008, VEIGA, VEIGA e DUCLÓS, 2010, ROMUALDO, BAPTISTA e VIEIRA, 2010, CHEN, 2011), apesar de múltiplos critérios serem desejáveis na avaliação prática das técnicas de previsão (LEITCH e TANNER, 1991, YOKUM e ARMSTRONG, 1995). Grande parte dos trabalhos científicos publicados utiliza a acuracidade como parâmetro de escolha do modelo de previsão, mas poucos analisam a influência dos erros de previsão no gerenciamento da cadeia de suprimentos ou no desempenho empresarial (ACAR E GARDNER, 2012). Além de poucas publicações, a literatura também revela resultados contraditórios. Alguns estudos demonstram que os erros de previsão apresentam significante impacto nos custos totais, na programação da produção e no nível de serviço prestado (XIE, LEE e ZHAO, 2004), outros demonstram resultados totalmente opostos (PRICE e SHARP, 1985, HO e IRELAND, 1998, COELHO, SANTOS e COSTA JR, 2008).

No mundo altamente competitivo de hoje, o balanço entre falha e sucesso de uma organização é tão fino que a sobrevivência depende significativamente da qualidade da informação que molda as decisões gerenciais. O debate acerca do método quantitativo ideal para previsão de demanda de produtos alimentícios e do impacto financeiro decorrente dos erros de previsão ainda não atingiu um consenso. Este fato estimula a continuidade de pesquisa nesta área do conhecimento.

\section{METODOLOGIA UTILIZADA}

A indústria em estudo é uma empresa de representatividade no mercado de alimentos no Brasil sendo composta por unidades industriais e unidades de negócios concentradas nas principais regiões do país. Seu portfólio é composto por uma grande diversidade de mix dentro de cada divisão. Este trabalho avaliou apenas uma das cinco regiões brasileiras que compõem a unidade de negócios de produtos alimentícios. Foram utilizados dados históricos da demanda abrangendo o período de 2004 a 2008. O período de análise foi selecionado mediante a necessidade de se 
obter, no mínimo, 60 séries temporais a fim de possibilitar a identificação de padrões como nível, tendência e sazonalidade. Trata-se de um estudo de caso descritivo expost facto com corte temporal seccional.

Para facilitar a análise e para aumentar sua relevância gerencial, grupos de produtos semelhantes foram agregados e analisados conjuntamente. Como a escolha do nível apropriado de agregação depende do processo de tomada de decisão que a previsão espera suportar (ZOTTERI, KALCHSCHMIDT e CANIATO, 2005), o critério para agregação foi selecionado com base nas características intrínsecas dos produtos, reunindo-os por caracteres de similaridade entre si. Pela agregação dos dados foram formados 5 grupos de produtos: (i) grupo A: produtos que representam $70 \%$ de todo o volume de vendas da empresa. Esta linha é composta por um total de 59 SKUs (Stock Keeping Units); (ii) grupo B: produtos que representam 10,5\% de todo volume de vendas da empresa. Esta linha é composta por 9 SKUs; (iii) grupo C: produtos que representam 8,5\% de todo volume de vendas da empresa. Esta linha é composta de 13 SKUs; (iv) grupo D: produtos que representam $10 \%$ de todo o volume de vendas da empresa. Esta linha é composta de um total de 8 SKUs; (v) grupo E: são produtos que representam $1 \%$ de todo o volume de vendas da empresa. Esta linha é composta por 2 SKUs.

A análise quantitativa dos dados de pesquisa está dividida em três etapas de acordo com os objetivos propostos. A primeira etapa consiste em comparar a acurácia de métodos lineares de previsão de demanda e a rede neural em relação ao atual método utilizado pela empresa em estudo. A segunda etapa avalia a responsividade de atendimento da demanda, informação necessária para que a terceira etapa de pesquisa possa determinar a influência dos erros de previsão no desempenho financeiro da empresa.

\subsection{Escolha do modelo quantitativo de maior acurácia}

Para a série temporal histórica de cada grupo de produtos foram aplicados cinco métodos quantitativos de previsão de demanda: quatro modelos lineares: (i) suavização exponencial simples (SES), (ii) modelo de Holt (MH), (iii) modelo de Winter (MW) e (iv) modelo auto-regressivo integrado de médias móveis (ARIMA), e um modelo não-linear, as redes neurais feedforward treinadas com algoritmo de 
Levenberg-Marquadt. Os resultados foram comparados com os valores reais da demanda a fim de escolher o modelo de maior acurácia para cada grupo de produto.

A acurácia da previsão de demanda foi medida pela diferença entre a previsão para o período $t$ e a demanda real no período $t$ (CHOPRA e MEINDL, 2003). Assim como em outros trabalhos científicos (SWANSON e WHITE, 1997, CHU e ZHANG, 2003, PAO, 2006, CHEN, 2011), esta variável foi operacionalmente calculada com base no Erro Percentual Absoluto Médio (MAPE), expresso matematicamente pela Equação 1:

$$
\begin{aligned}
M A P E_{n} & =\frac{\sum_{t=1}^{n}\left|\frac{E_{t}}{D_{t}}\right| \text { ivo }}{n} \\
\left|\mathrm{E}_{t}\right| & =\text { valor absoluto do erro no período } t \\
\left|\mathrm{D}_{t}\right| & =\text { valor absoluto de demanda real no período } t \\
n & =\text { todos os períodos. }
\end{aligned}
$$

\subsection{Responsividade de atendimento da demanda}

A etapa 2 utilizou o método quantitativo de previsão de demanda de maior grau de acurácia (com base no menor valor do MAPE) de cada um dos cinco grupos de produtos para prosseguir na análise. Foi utilizado o índice de responsividade como indicador do nível de serviço oferecido ao consumidor, assim como do custo de oportunidade em resposta à demanda. A responsividade de atendimento da demanda pode ser definida como o percentual da demanda satisfeita diretamente no ponto de venda durante um ciclo de reabastecimento (ZENG, 2000). O índice de responsividade de atendimento da demanda também é denominado de fill rate (FR) e foi calculado como demonstrado na Equação 2:

$$
\mathrm{FR}=1-\frac{\text { mümero de stockouts esperados por ciclo }}{\text { número de unidades exi gidas por ciclo }}
$$

\subsection{Desempenho financeiro}

Na etapa 3, os resultados obtidos na etapa 2 foram, por fim, comparados com o fill rate da empresa em estudo para determinar a diferença entre demanda atendida e demanda real. O desempenho financeiro é dependente da diferença 
entre as possíveis receitas e custos (LIMA, 2003). Desta forma, o desempenho financeiro foi mensurado neste trabalho como custo de oportunidade através do fill rate, expresso matematicamente pela Equação 3.

$$
\begin{aligned}
& \mathrm{FR}=1-\frac{\alpha \mathrm{G}_{\mu}[K]}{Q} \\
& \mu=\text { média, } \\
& \alpha=\text { desvio padrão, } \\
& K=\text { fator de segurança, } \\
& \alpha \mathrm{G}_{\mu}(K)=\text { função de unidade padrão perdida (stockout), } \\
& Q=\text { pedido quantitativo. }
\end{aligned}
$$

A limitação deste estudo se deve à qualidade dos dados fornecidos pela empresa em estudo, bem como às diversas variáveis que compõem o referido negócio. Informações mercadológicas sobre o histórico de dados coletados não foram considerados na análise. Desta forma, determinadas condições tais como promoções, campanhas e ações da concorrência podem ter interferido em alguns resultados mensais na variação da demanda. Do mesmo modo, este estudo não considerou possíveis restrições na capacidade de produção da organização em estudo nem tamanho mínimo de lote para comercialização. Na próxima seção serão apresentados os resultados obtidos pela aplicação da metodologia descrita acima.

\section{RESULTADOS E ANÁLISE DOS DADOS}

Esta seção encontra-se dividida em três etapas para facilitar a análise e a interpretação dos dados. A primeira etapa demonstra uma avaliação prévia da série temporal histórica em estudo. A segunda etapa avalia a acurácia dos métodos lineares de previsão de demanda e das redes neurais em relação ao atual método utilizado pela empresa em estudo. Por fim, a terceira etapa determina a influência dos erros de previsão no desempenho financeiro da empresa.

\subsection{Análise prévia dos dados}

Para a aplicação dos métodos de previsão, é imprescindível analisar os dados em estudo com a finalidade de identificar os padrões ou fatores componentes da curva obtida, tais como tendência, sazonalidade, variações irregulares e variações randômicas. Estes padrões de comportamento podem interferir na acuracidade dos 
métodos quantitativos empregados e, por isso, devem ser conhecidos a fim de serem considerados na análise dos resultados.

Figura 1 - Análise da série temporal histórica dos produtos do grupo A entre 2004 e 2008

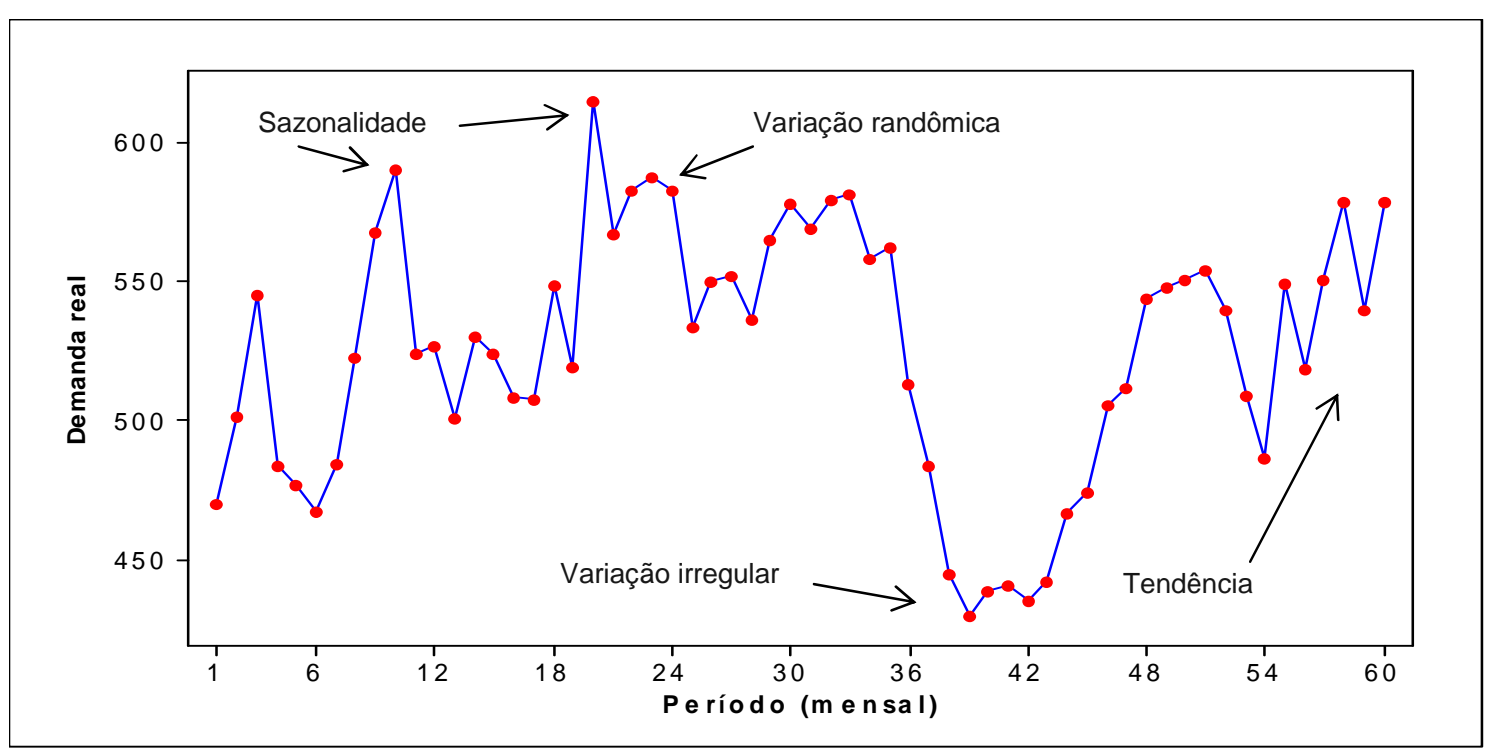

As Figuras 1 e 2 representam graficamente a série de demanda agregada para os grupos de produto A, B, C, D e E. Os produtos do grupo A (Figura 1) foram avaliados em separado para facilitar a escolha da escala horizontal (demanda em toneladas) já que representam $70 \%$ de todo volume de vendas da empresa. Tanto a Figura 1 quanto a Figura 2 demonstram que as séries temporais históricas em estudo apresentam tendência, sazonalidade, variações randômica e irregular. 
Figura 2 - Análise da série temporal histórica dos grupos de produto B, C, D e E entre 2004 e 2008

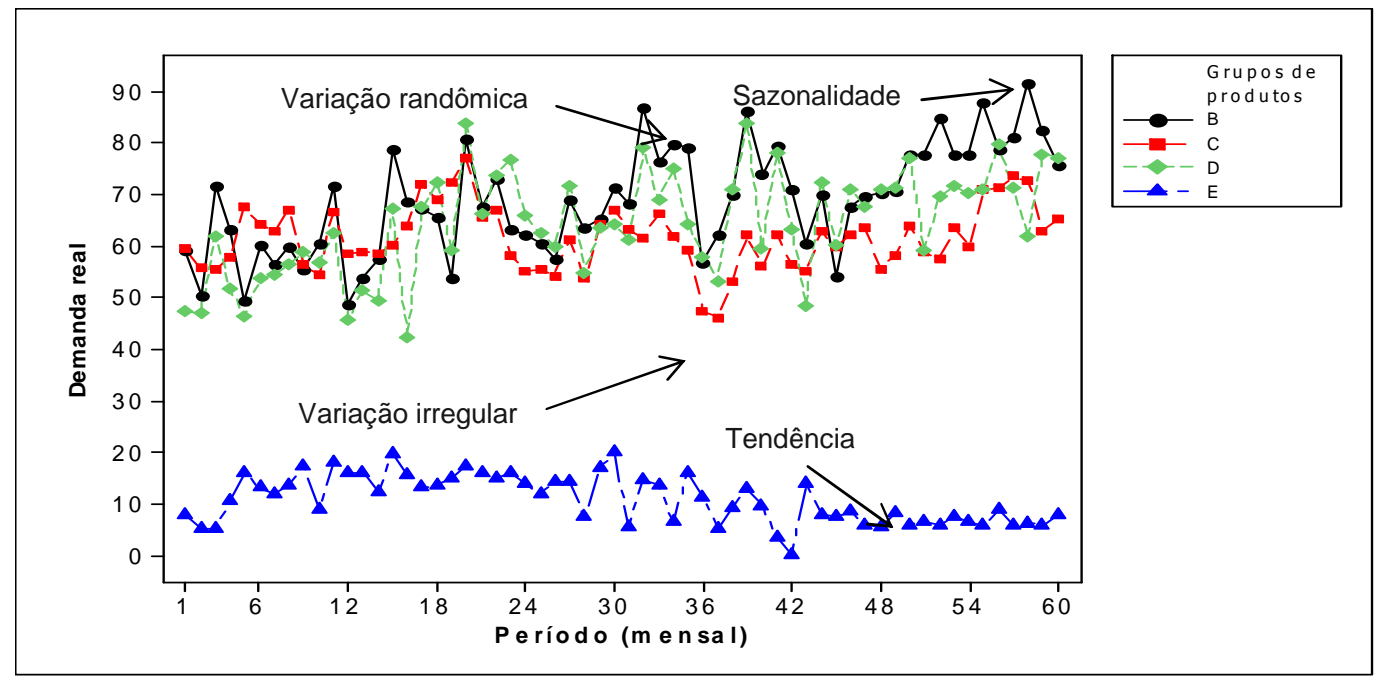

\subsection{Comparação dos métodos de previsão da demanda}

A previsão de demanda da empresa em estudo utiliza o método de médias móveis aliado a uma valorização qualitativa, que pode oscilar de acordo com as ações de mercado. A previsão tem como base o software Microsoft Excel ${ }^{\circledR}$, com utilização de planilhas eletrônicas semanais para a composição da previsão mensal. Nestas, avalia-se a média de demanda das últimas 08 semanas e a máxima no período. A média aritmética entre as semanas que compõe o mês corrente é utilizada para realizar a previsão do mês seguinte. Além da análise quantitativa, o analista de previsão possui autonomia para fazer as alterações necessárias em função da necessidade da cada setor de vendas.

Dados da literatura demonstram que o método de médias móveis traz resultados satisfatórios apenas quando a demanda não apresenta comportamentos de tendência e/ou sazonalidade (DELURGIO, 1998, MAKRIDAKIS, WHEELWRIGHT e HYNDMAN, 1998, CHOPRA e MEINDL, 2003, SOUZA, SAMOHYL e MIRANDA, 2008). Como este não é o caso da indústria de alimentos, este método apresenta diversas limitações que podem impactar nos custos totais, na programação da produção e no nível de serviço prestado pela empresa.

Para os modelos SES, MH e MW, os dados de ajustes foram gerados pelo programa estatístico NNQ-STAT ${ }^{\circledR}$ para 12 períodos (sazonal). Para o modelo 
ARIMA, os ajustamentos da previsão foram feitos pelo programa estatístico NCSS ${ }^{\circledR}$ (2007). Para este último, foram considerados os seguintes parâmetros de ajustamento ( $p, d, q)$ em função do menor valor de MAPE: produtos do grupo A $(1,0,1)$, produtos do grupo $B(1,0,1)$, produtos do grupo $C(1,1,2)$, produtos do grupo D $(1,0,1)$, produtos do grupo $E(2,0,2)$.

Para aplicação do método RN utilizou-se o software MATLAB ${ }^{\circledR}$ (2008) para implementação das rotinas computacionais de estimação dos modelos. Foram utilizadas as séries atrasadas em 2, 3, 4, 5, 6, 12, 24 e 48 meses, seguindo-se uma ordem lógica de descarte de piores resultados. Para cada experiência, foi necessário procurar a melhor configuração, ou seja, testar diversos números de neurônios na camada intermediária. A análise dos resíduos possibilitou verificar a adequação do modelo aos dados e a escolha da melhor rede. Os parâmetros foram selecionados com base no menor valor de MAPE.

Para todos os grupos de produtos, os ajustes da previsão foram realizados do ano de 2004 a 2007. As observações do comportamento passado foram então analisadas por modelos matemáticos para extrapolar a série temporal para o ano de 2008. Os resultados foram comparados com os valores da demanda real. As Figuras 3, 4, 5, 6 e 7 apresentam os resultados para os grupos de produto A, B, $\mathrm{C}, \mathrm{D}$ e $\mathrm{E}$, respectivamente.

Figura 3 - Previsão de demanda dos produtos do grupo A para o ano de 2008

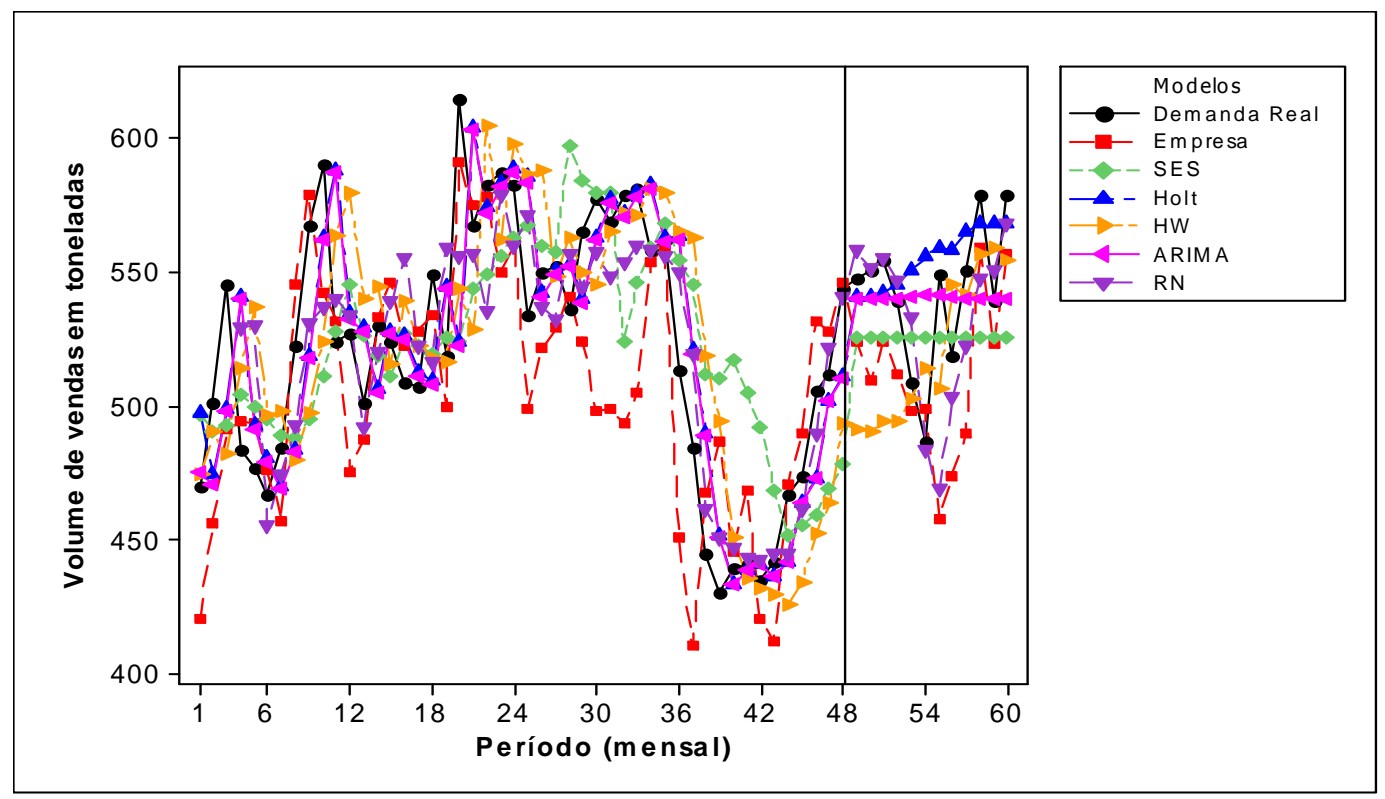


A Figura 3 apresenta graficamente os resultados obtidos pela aplicação dos modelos de previsão estudados para os produtos do grupo A. Para este grupo de produtos, o modelo RN apresentou maior grau de acurácia com valor de MAPE de 3,40. O modelo de previsão adotado pela empresa apresentou menor grau de acurácia em comparação a todos os modelos de previsão empregados na análise, com MAPE de 6,15.

A Figura 4 apresenta graficamente os resultados obtidos pela aplicação dos modelos de previsão estudados para os produtos do grupo B. Para este grupo de produtos, o modelo ARIMA apresentou maior grau de acurácia com valor de MAPE de 7,52. O modelo da empresa apresentou grau de acurácia superior ao modelo SES e resultado inferior aos demais modelos.

Figura 4 - Previsão de demanda dos produtos do grupo B para o ano de 2008

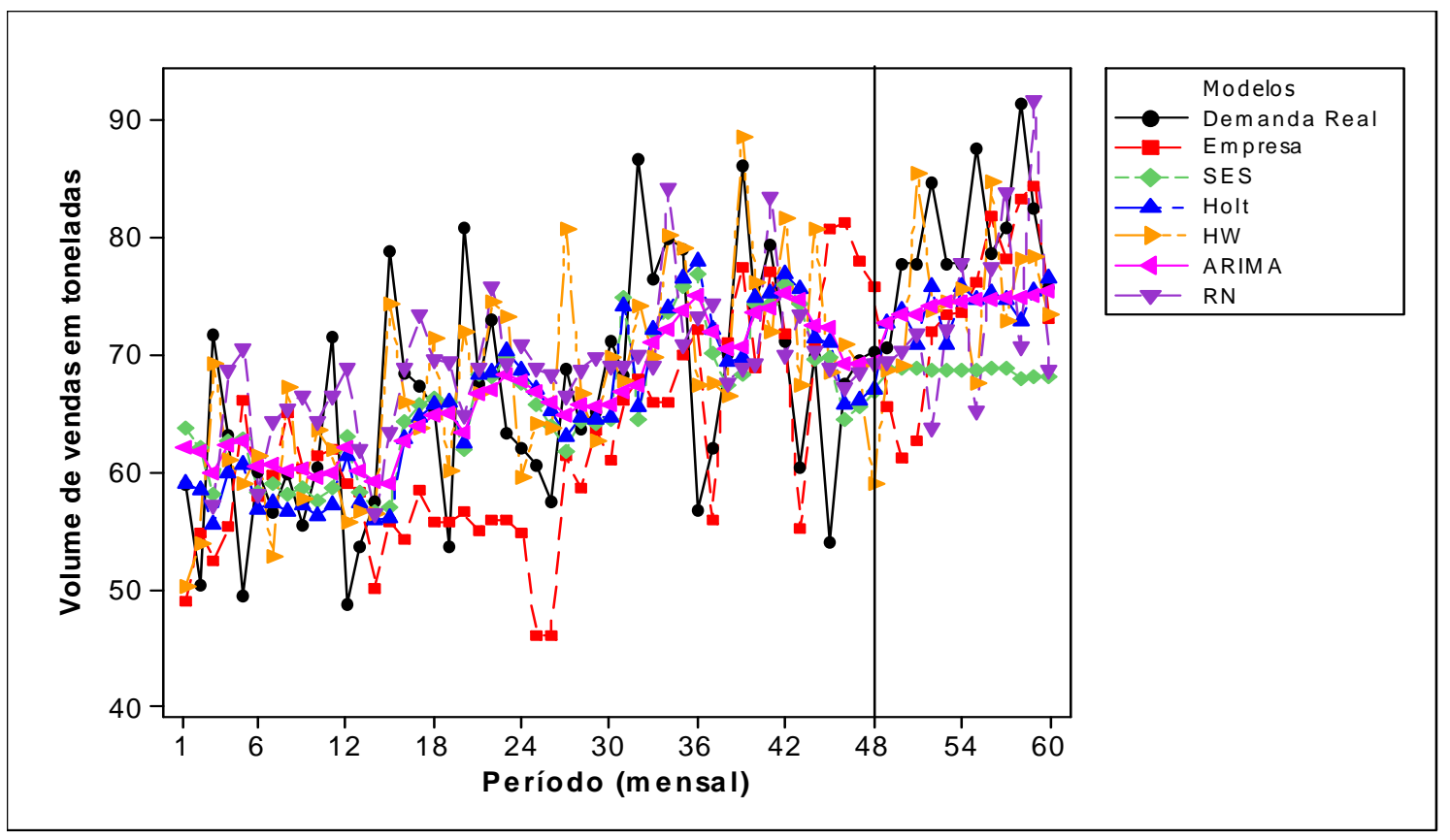

Os resultados para os produtos do Grupo C são apresentados pela Figura 5. Para este grupo de produto, o modelo RN apresentou maior grau de acurácia com valor de MAPE de 5,20. O modelo da empresa apresentou grau de acurácia superior aos modelos SES, MH e ARIMA. 
Figura 5 - Previsão de demanda dos produtos do grupo C para o ano de 2008

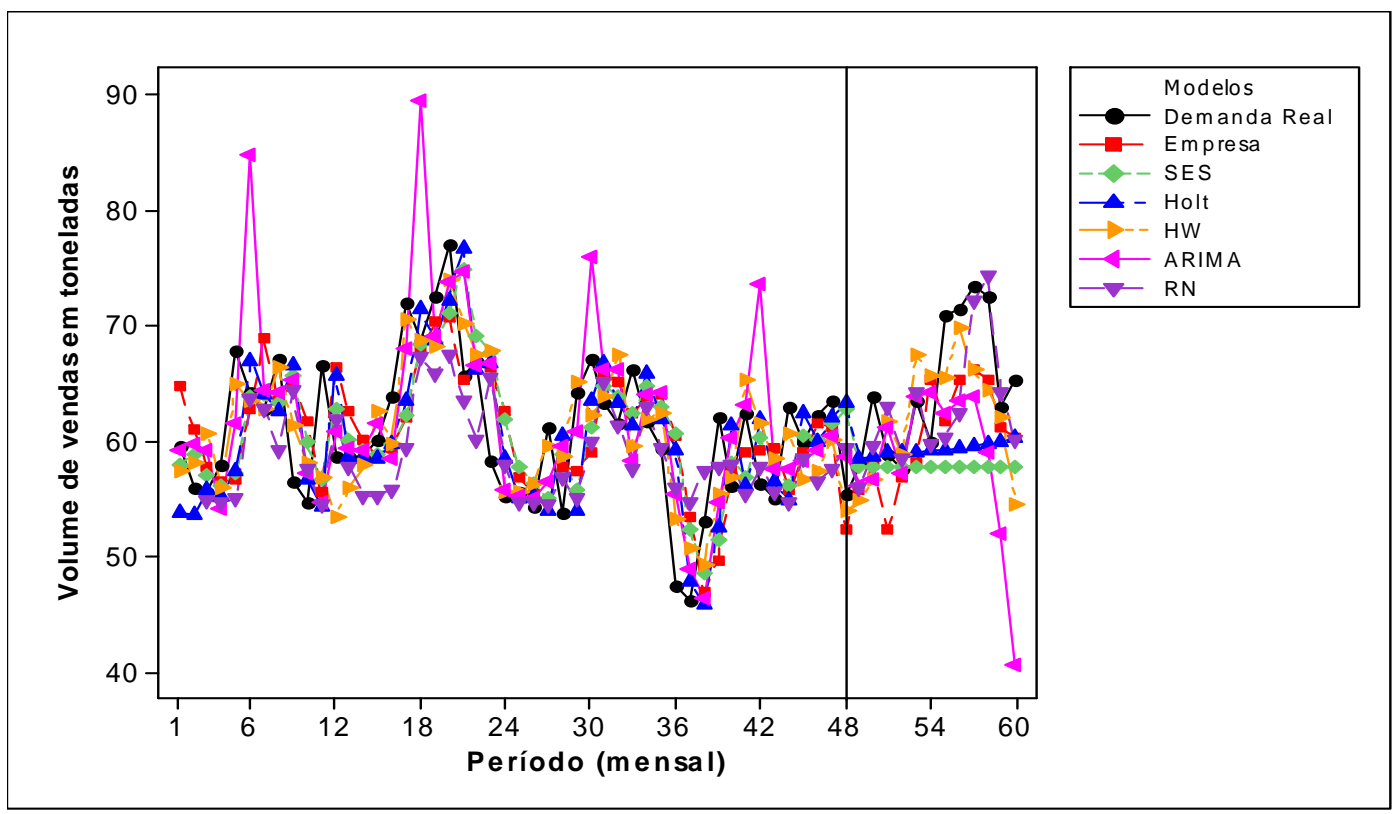

Os resultados para os produtos do Grupo D são apresentados pela Figura 6. Para este grupo de produto, o $\mathrm{MH}$ apresentou maior grau de acurácia com valor de MAPE de 6,44. O modelo da empresa apresentou grau de acurácia superior ao MW e desempenho inferior aos demais.

Figura 6 - Previsão de demanda dos produtos do grupo D para o ano de 2008

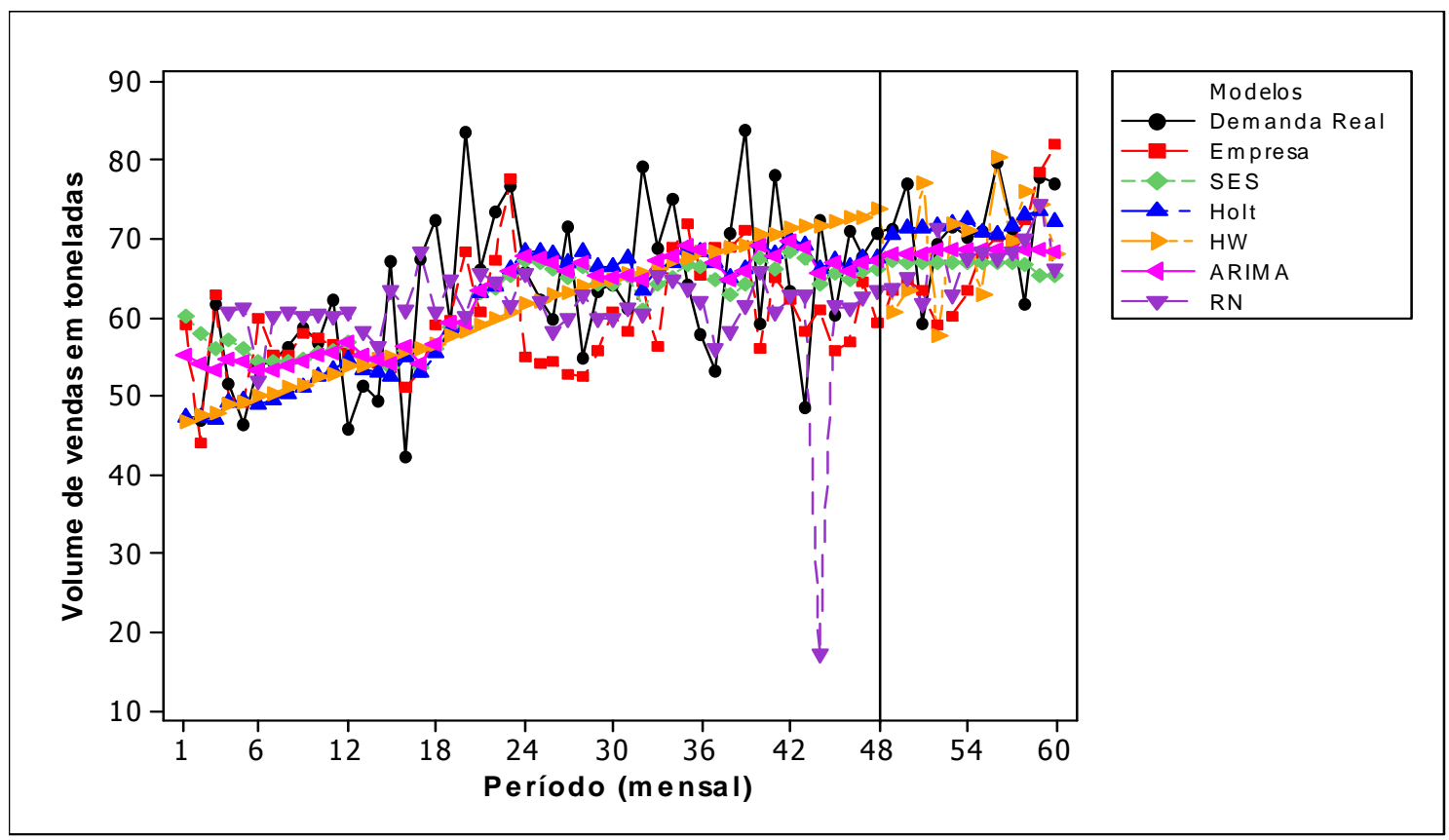

Os resultados para os produtos do Grupo E são apresentados pela Figura 7. Para este grupo de produtos, o modelo ARIMA apresentou maior grau de acurácia 
com valor de MAPE de 11,49. O modelo adotado pela empresa apresentou grau de acurácia superior aos modelos, SES, MH e MW.

Figura 7 - Previsão de demanda dos produtos do grupo E para o ano de 2008

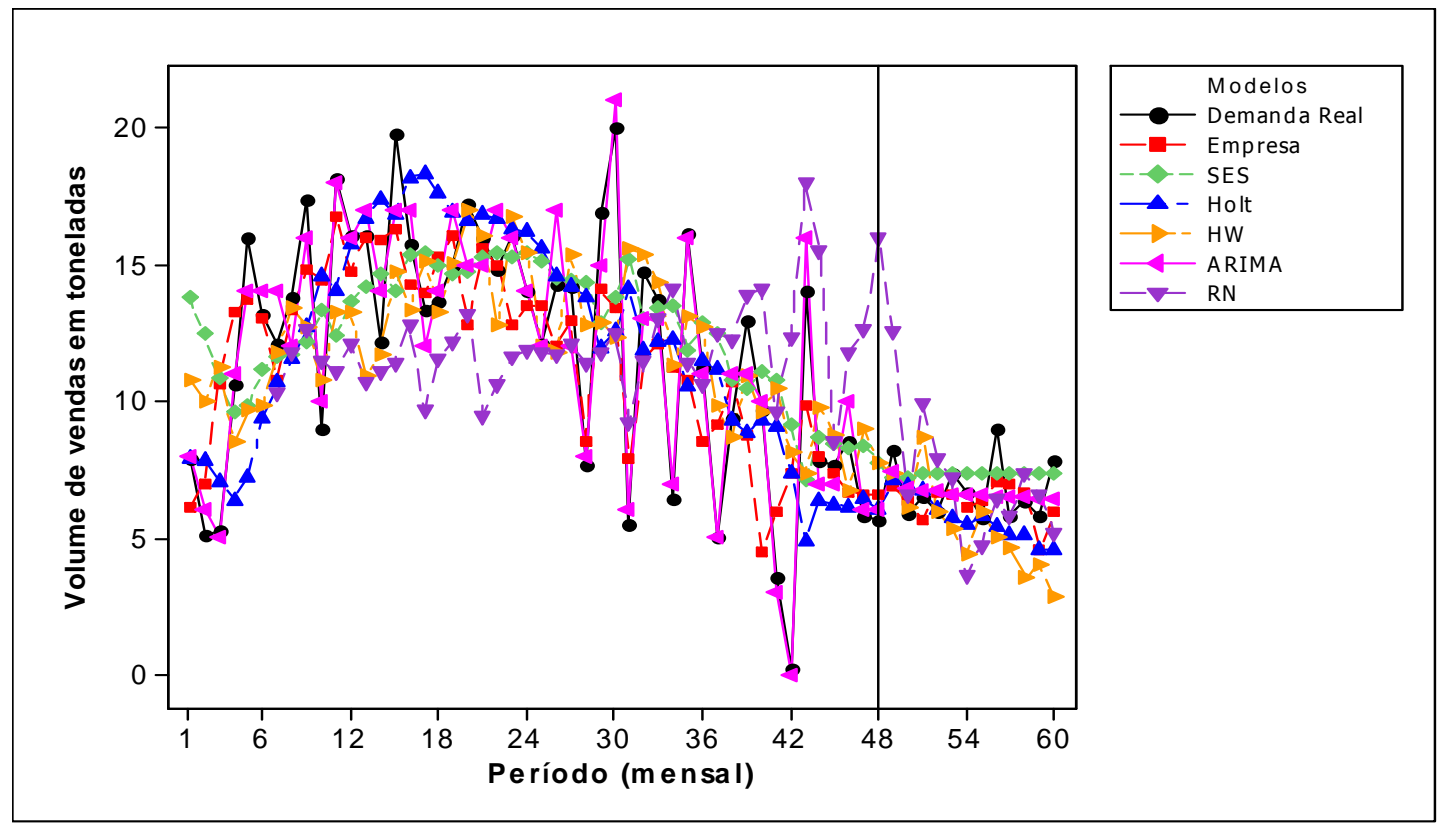

Tabela 1 - Grau de acurácia para diversos modelos de previsão de demanda para os grupos de produto $A, B, C, D$ e E, segundo o MAPE

\begin{tabular}{lccccc}
\hline \multicolumn{1}{c}{ Modelos } & Grupo A & Grupo B & Grupo C & Grupo D & Grupo E \\
\hline Empresa & 6,15 & 9,13 & 7,93 & 9,81 & 14,29 \\
SES & 4,88 & 14,20 & 10,34 & 9.50 & 17,18 \\
MH & 4,16 & 8,00 & 8,56 & $\mathbf{6 , 4 4}$ & 17,67 \\
MW & 6,14 & 8,91 & 7,43 & 11,26 & 26,42 \\
ARIMA & 3,71 & $\mathbf{7 , 5 2}$ & 11,44 & 7,97 & $\mathbf{1 1 , 4 9}$ \\
RN & $\mathbf{3 , 4 0}$ & 10,42 & $\mathbf{5 , 2 0}$ & 8,75 & 25,56 \\
\hline
\end{tabular}

A Tabela 1 resume os resultados de MAPE obtidos pela aplicação dos cinco modelos de previsão de demanda aos grupos de produtos A, B, C, D, e E. Em suma, pode-se dizer que para os grupos de produto $A$ e $\mathrm{C}$, o modelo $\mathrm{RN}$ apresentou maior grau de acurácia, para os produtos do grupo $B$ e E, o modelo de ARIMA apresentou menor MAPE, e para os produtos do grupo $\mathrm{D}$, o $\mathrm{MH}$ obteve maior desempenho preditivo. Em função destes resultados, foram adotados os modelos RN, ARIMA e MH para o cálculo do índice de responsividade da demanda (fill rate). 


\subsection{Responsividade da demanda e desempenho financeiro}

Tabela 4 - Análise do desempenho financeiro da empresa em função dos erros de previsão de demanda

\begin{tabular}{|c|c|c|c|c|c|c|c|c|c|c|c|c|}
\hline \multicolumn{2}{|c|}{$\begin{array}{l}\text { Demanda Real } \\
\text { em toneladas }\end{array}$} & \multicolumn{5}{|c|}{$\begin{array}{c}\text { Vendas realizadas pelo modelo de } \\
\text { previsão da Empresa }\end{array}$} & \multicolumn{6}{|c|}{$\begin{array}{l}\text { Previsão realizada pelo modelo de melhor } \\
\text { desempenho com base no MAPE }\end{array}$} \\
\hline 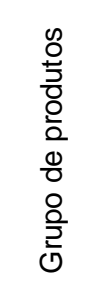 & $\begin{array}{l}\frac{0}{0} \\
\frac{\pi}{0} \\
\frac{\pi}{10} \\
0 \\
0\end{array}$ & $\begin{array}{l}\frac{0}{\pi} \\
0 \\
\frac{\pi}{0} \\
0 \\
0\end{array}$ & $\begin{array}{l}\frac{\widehat{D}}{\tilde{0}} \\
\frac{0}{0} \\
\frac{0}{0}\end{array}$ & 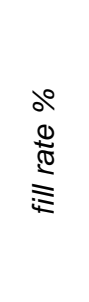 & 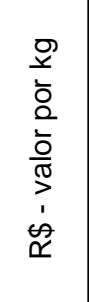 & 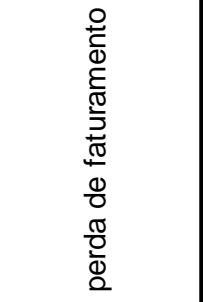 & $\begin{array}{l}\frac{0}{0} \\
\frac{\pi}{0} \\
\frac{\pi}{10} \\
0 \\
0\end{array}$ & $\begin{array}{l}\frac{\widehat{D}}{\tilde{0}} \\
\frac{0}{0} \\
\frac{0}{0}\end{array}$ & 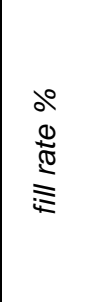 & 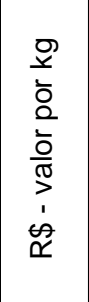 & 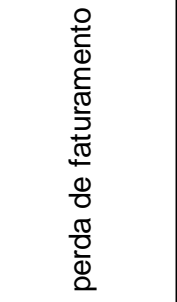 & 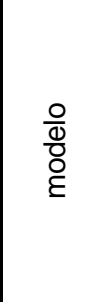 \\
\hline A & 6503,26 & 6126,26 & 377,00 & 94,20 & 6,37 & $2.401 .490,00$ & 6390,25 & 113,01 & 98,26 & 6,37 & $719.873,70$ & RN \\
\hline$B$ & \begin{tabular}{|l|}
963,28 \\
\end{tabular} & 885,29 & 77,99 & 91,90 & \begin{tabular}{|l|}
8,27 \\
\end{tabular} & $644.977,30$ & 892,50 & $\begin{array}{l}70,78 \\
\end{array}$ & 92,65 & \begin{tabular}{|l|}
8,27 \\
\end{tabular} & $585.350,60$ & ARIMA \\
\hline C & \begin{tabular}{|l|}
778,56 \\
\end{tabular} & 726,50 & 52,06 & 93,31 & 9,69 & $504.461,40$ & 754,29 & 24,27 & 96,88 & 9,69 & $235.176,30$ & $\mathrm{RN}$ \\
\hline $\bar{D}$ & \begin{tabular}{|l|}
858,28 \\
\end{tabular} & 815,42 & 42,86 & 95,01 & 9,80 & $420.028,00$ & 833,30 & 24,98 & 97,09 & 9,80 & $244.804,00$ & Holt \\
\hline$E$ & 81,09 & 75,74 & 5,35 & 93,40 & 15,00 & $80.250,00$ & 79,90 & 1,19 & 98,53 & 15,00 & $17.850,00$ & ARIMA \\
\hline
\end{tabular}

Os cálculos de responsividade da demanda e desempenho financeiro abrangeram um período de 12 meses (01/01/2008 a 31/12/2008). Em suma, os resultados demonstram que o modelo ARIMA estima um atendimento da demanda que varia de $92,65 \%$ a $98,53 \%$ para os produtos dos grupos B e E. O modelo RN estima um atendimento da demanda de $96,88 \%$ a $98,26 \%$ para os produtos do grupo A e C. O modelo de Holt estima um atendimento da demanda de $97,09 \%$ para os produtos do grupo $D$.

De um modo geral, pode-se afirmar que todos os modelos estudados apresentaram uma previsão potencialmente boa, tendo em vista o valor de MAPE em torno de $10 \%$. Os resultados demonstrados para os grupos de produtos $A, B, C$, $D$ e $E$ confirmam que a acuracidade da previsão tem influência direta no nível de serviço oferecido ao consumidor e, por conseguinte, no desempenho financeiro da organização como um todo. Para os produtos do grupo A, a empresa deixa de abastecer o mercado/demanda em 377,00 toneladas, com perda de um faturamento aproximado de $R \$ 2.401 .490,00$. Para os produtos do grupo $B$, ao utilizar o método atual de previsão, a empresa deixa de abastecer o mercado/demanda em 77,99 toneladas, com perda de um faturamento aproximado de $\mathrm{R} \$ 644.977,30$. Para os produtos do grupo C, esta ruptura chega a 52,06 toneladas, com perda de faturamento aproximado de $\mathrm{R} \$ 504.461,40$. Para os produtos do grupo $D$, esta deficiência chega em 42,86 toneladas, com perda de um faturamento aproximado de 
$\mathrm{R} \$ 420.028,00$. Para produtos do grupo $\mathrm{E}$, a ruptura é de 5,35 toneladas, com perda de faturamento aproximado de $R \$ 80.250,00$. O impacto financeiro total referente aos erros de previsão de demanda para a empresa é de aproximadamente $R \$$ 4.051.206,70 (6,05\%) anuais. Se a empresa empregasse o modelo RN para os produtos dos grupos A e C, o modelo ARIMA para os produtos do grupo B e $E$, o modelo de Holt para os produtos do grupo D, o faturamento poderia ser aumentado em uma média aproximada de $\mathrm{R} \$ 2.248 .377,48$ anuais.

\section{CONSIDERAÇÕES FINAIS}

No mundo corporativo, passado e futuro determinam a natureza das tomadas de decisão. Quando baseadas em fatos futuros, as decisões precisam ser apoiadas em processos com elevado grau de acurácia, o que pode determinar redução de custos operacionais de produção e logística e ampla disponibilidade de produto oferecidos aos clientes. Nesse sentido, as medidas de erros de previsão são bastante úteis já que visam a melhoria contínua do processo de previsão. Desta forma, o presente trabalho comparou a acurácia de quatro métodos lineares de previsão de demanda e de redes neurais em relação à atual metodologia utilizada pela empresa em estudo. Em uma segunda etapa, os resultados desta análise serviram como subsídio para avaliar a influência dos erros de previsão de demanda no desempenho financeiro da empresa.

Para o presente trabalho, dois grupos de produtos apresentaram melhor acuracidade com o método de $\mathrm{RN}$ (grupos A e C), dois grupos com o modelo ARIMA (grupos B e E) e um grupo de produtos com o $\mathrm{MH}$. É importante ressaltar ainda que, para o grupo A, a diferença do resultado de MAPE entre o modelo $\operatorname{ARIMA}(3,71)$ e $\mathrm{RN}(3,40)$ foi de apenas 0,31 , o que representa um aumento pouco significativo no desempenho financeiro da empresa. Em contrapartida, para o grupo $\mathrm{C}$, a diferença do resultado de MAPE entre o modelo ARIMA $(11,44)$ e $R N(5,20)$ foi de 6,24 , o que geraria um ganho substancial adicional no faturamento da empresa em estudo.

Em suma, pode-se concluir que não há uma única resposta para o modelo de previsão ideal para a unidade de negócios de alimentos já que dos cinco grupos de produto testados, três apresentaram resultados satisfatórios para os modelos lineares $(B, D$ e $E)$ e dois para a $R N(A$ e $C)$. A comparação de acuracidade e 
desempenho financeiro entre modelos lineares e $\mathrm{RN}$ demonstram que outros parâmetros deveriam ser avaliados para validar os ganhos reais pela escolha de uma metodologia em detrimento a outras.

\section{CONCLUSÕES}

A acuracidade de um modelo de previsão é considerado o critério mais importante na seleção do método quantitativo de análise. Entretanto, dentro de uma perspectiva prática, outros critérios também deveriam ser avaliados tais como interpretação, uso, flexibilidade, empregabilidade dos dados disponíveis e implementação do processo (YOKUM e ARMSTRONG, 1995). Isto é particularmente importante para empresas que possuem portfólio variado e seleção de diferentes métodos ideais de previsão para cada grupo de produtos. Desta forma, sugere-se para trabalhos futuros avaliar a acuracidade de métodos de previsão combinados a fim de atender ao comportamento variado das séries temporais em estudo e, ao mesmo tempo, a comparação das metodologias empregadas em termos de facilidade de uso, facilidade de interpretação e flexibilidade. É importante considerar que, apesar de tantos benefícios, modelos complexos de previsão de demanda podem não ser viáveis para todas as organizações.

A indústria de alimentos constitui um setor representativo na economia nacional e, como em outros segmentos, precisa planejar sua produção, cujos produtos são sensíveis à sazonalidade de oferta e de demanda, perecíveis e de acentuada diversidade (QUEIROZ e CAVALEIRO, 2003, VEIGA, 2009). O mercado de alimentos, por sua vez, apresenta planos de vendas irregulares (flutuações sazonais), demanda influenciada por muitos fatores, uso frequente de atividades promocionais, muitos intermediários no processo, diversidade de clientes e alto nível de serviço exigido pelo consumidor. A associação entre as incertezas causadas pelas características intrínsecas de produtos alimentícios e as características extrínsecas relacionadas ao mercado, torna o sistema de previsão de demanda viável e necessário para o planejamento estratégico e competitivo em uma empresa de alimentos (MEIJDEN; NUNEN e RAMONDT, 1994).

$\mathrm{Na}$ literatura sobre previsão, é bem estabelecido que nenhum modelo quantitativo seria o ideal para todas as situações sob qualquer circunstâncias. 
Portanto, o "melhor" modelo de previsão do mundo real deveria ser aquele que possui robustez e acuracidade por um longo horizonte temporal e que, ao mesmo tempo, ofereça confiança e facilidade para que os usuários possam utilizá-lo repetidas vezes. Para a indústria de alimentos, os resultados ainda não demonstram consenso, fato este que exige novos estudos investigativos específicos para cada condição de estudo.

\section{REFERÊNCIAS}

ACAR, Y.; GARDNER, J. E. S. Forecasting method selection in a global supply. International Journal of Forecasting. Turquia, 2012. doi:10.1016/.i.jforecast.2011.11.003

ALI, M. M.; BOYLAN, J. E.; SYNTETOS, A. A. Forecast errors and inventory performance under forecast information sharing. International Journal of Forecasting, Reino Unido, 2011. doi:10.1016/j.ijforecast.2010.08.003.

BARBOSA, M. A.; WANKE, P. Combinação ou competição de previsões: um estudo de caso nos fretes do agronegócio. Logística e Supply Chain Management, Brasil, n. 17, ano III, p. 68-73, jul./ago. 2010.

BOX, G. E. P.; JENKINS, G. M.; REINESL, G. C. Time series analysis: forecasting and control. 3. ed. New Jersey: Prentice-Hall, 1994. 598 p.

CALÔBA, G. M. CALÔBA, L. P.; SALIBY, E. Cooperação entre redes neurais artificiais e técnicas "clássicas" para previsão de demanda de uma série de vendas de cerveja na Austrália. Pesquisa Operacional, Rio de Janeiro, v. 22, n .3, p. 345358, 2002.

CHELANI, A. B.; DEVOTTA, S. Air quality forecasting using a hybrid autoregressive and nonlinear model. Atmospheric environment, Índia, v. 40, p. 1774-1780, 2006.

CHEN, K-Y. Combining linear and nonlinear model in forecasting tourism demand. Expert Systems with applications. Taiwan, 2011. doi:10.1016/j.eswa.2011.02.049.

CHOPRA, S.; MEINDL, P. Gerenciamento da cadeia de suprimentos: estratégia, planejamento e operações. São Paulo: Pearson Prentice Hall, 2001. 465 p.

CHU, C-W.; ZHANG, G. P. A comparative study of linear and nonlinear models for aggregate retail sales forecasting. International Journal of Production

Economics, Estados Unidos, v. 86, p. 217-231, 2003. 
CO, H. C.; BOOSARAWONGSE, R. Forecasting Thailand's rice export: statistical techniques vs. artificial neural networks. Computers \& Industrial Engineering, Estados Unidos, v. 53, p. 610-627, 2007.

COELHO, L. S.; SANTOS, A. A. P.; COSTA JR, N. C. A. Can we forecast Brazilian exchange rates?: empirical evidences using computational intelligence and econometric models. Gestão e Produção, Brasil, v. 15, n. 3, p. 1-14, 2008.

DELURGIO, S. A. Forecasting principles and applications. Boston: Irwin McGrawHill, 1998. $802 \mathrm{p}$.

FARIA, E. L.; ALBUQUERQUE, M. P.; ALFONSO, J. L. G.; ALBUQUERQUE, M. P.; CAVALCANTE, J. T. P. Previsão de séries temporais utilizando métodos estatísticos. 2008. Disponível em: <http//www.cbpfinder.cbpf.br/publication_pdfs>. Acesso em: 14 jun. 2009.

GUTIERREZ-ESTRADA, J. C.; PEDRO-SANZ, E.; LÓPEZ-LUQUE, R.; PULIDOCALVO, I. Comparison between traditional methods and artificial neural networks for ammonia concentration forecasting in na eel (Anguilla Anguilla L.) intensive rearing system. Aquacultural Engineering, Espanha, v. 31, n. 3/4, p. 183-203, 2004.

HANN, T. H.; STEURER, E. Much ado about nothing? Exchange rate forecasting: neural networks vs. linear models using monthly and weekly data. Neurocomputing, Alemanha, v. 10, p. 323-339, 1996.

HAYKIN, S. Redes neurais: princípios e prática. 2. ed. Porto Alegre: Bookman, 2001.

HERAVI, S.; OSBORN, D. R.; BIRCHENHALL, C. R. Linear versus neural network forecast for European industrial production series. International Journal of Forecasting, Reino Unido, v. 20, p. 435-446, 2004.

HO, C. J.; IRELAND, T. C. Correlating MRP system nervousness with forecast errors. International Journal of Production Research, Estados Unidos, v. 36, p. 2289-2299, 1998.

HOLT, C. C. Author's retrospective on "Forecasting seasonal and trends by exponentially weighted moving averages". International Journal of Forecasting, Estados Unidos, v. 20, n. 1, p. 11-13, 2004.

KATATEPE, E.; ALCI, M. A New approach to fuzzy wavelet system modeling International Journal of Approximate Reasoning, Turquia, v. 40, p. 302-322, 2005. 
KERKKANEN, A.; KORPELA, J.; HUISKONEN, J. Demand forecasting errors in industrial context: measurement and impacts. International Journal Production Economics, Finlândia, v. 118, p. 43-48, 2009.

KOSKELA, T. Neural network methods in analysing and modelling time varying processes. 2003. $127 \mathrm{f}$.. Doctoral Thesis - Helsinki University of Technology, Department of Electrical and Communications Engineering, Finlândia, 2003

KOUTROUMANIDIS, T.; IOANNOU, K.; ARABATZIS, G. Predicting fuelwood prices in Greece with the use of ARIMA models, artificial neural networks and a hybrid ARIMA-ANN model. Energy Policy, Grécia, n. 37, p. 3627-3634, 2009.

KUO, R. J.; XUE, K. C. Fuzzy neural networks with application to sales forecasting. Fuzzy Sets and Systems, Taiwan, v. 108, p. 123-143, 1999.

LEITCH, G.; TANNER, E. Economic forecast evaluation: profits versus the conventional error measures. American Economic Reviews, Estados Unidos, v. 81, n. 3, p. 580-590, 1991.

LEVEMBERG, K.. A method for the solution of certain problems in least squares. Applied Mathematics, Estados Unidos, v. 2, p. 164-168, 1944.

LEVIS, A. A.; PAPAGEORGIOU, G. Customer demand forecasting via support vector regression analysis. Chemical Engineering Research and Design, Reino Unido, v. 83, n. A8, p. 1009-1018, 2005.

LIMA, M. Estoque: custo de oportunidade e impacto sobre os indicadores financeiros. 2003. Disponível em <http://www.ilos.com.br>. Acesso em: 14 jun. 2009.

MAKRIDAKIS, S.; WHEELWRIGHT, S. C.; HYNDMAN, R.J. Forecasting: methods and applications. 3.ed. New York: John Wiley \& Sons, 1998. 641 p.

MARTINEZ, R. O.; ZAMPROGNO, B. Comparação de algumas técnicas de previsão em análise de séries temporais. Revista Colombiana de Estatística, Colômbia, v.26, n. 2, p. 129-157, 2003.

MEIJDEN, V. D. L. H.; NUNEN, J. A. E. E. V.; RAMONDT, A. Forecasting: bridging the gap between sales and manufacturing. International Journal Production Economics, Holanda, v. 37, n. 1, p. 101-114, 1994.

MORETTIN, P. A.; TOLOI, C. M. C. Previsão de séries temporais. 2. ed. São Paulo: Atual, 1987. 436 p.

NEUMANN, D. Uma abordagem integrada para o planejamento de demanda de novos produtos. Logística e Supply Chain Management, Brasil, n. 15, p. 54-72, ano III Março \& Abril, 2010. 
OLIVEIRA, M. A.; BERGMANN, D. R.; FÁVERO, L. P. L. Previsão de retornos de ações de empresas dos setores financeiro e de alimentos por meio de redes neurais e modelos ARIMA-GARCH. In: SEMEAD, 9, 2007. Anais...São Paulo: FEA-USP, 2007. Disponível:

<http://www.ead.fea.usp.br/semead/10semead/sistema/resultado/trabalhosPDF/146. pdf>. Acesso em: 12 fevereiro 2011.

PAO, H-T. Comparing linear and nonlinear forecasts for Taiwan's electricity comsumption. Energy, Taiwan, v. 31, p. 2129-2141, 2006.

PRICE, D. H. R.; SHARP, J. A. Investigation of the impact of changes in demand forecasting method on the financial performance of an electricity supply undertaking. International Journal of Electrical Power \& Energy Systems, Reino Unido, v. 7, n. 3, p. 131-137, 1985.

QUEIROZ, A. Q.; CAVALHEIRO, D. Método de previsão de demanda e detecção de sazonalidade para o planejamento da produção de indústrias de alimentos. In: Encontro Nacional de Engenharia de Produção. Anais...Ouro Preto, MG, 2003. Disponível:

<http://www.abepro.org.br/biblioteca/ENEGEP2003_TR0101_0801.pdf> Acesso em: 10 abril 2012.

ROMUALDO, L. C. S.; BAPTISTA, E.; VIEIRA, D. R. Sistema Fuzzy-Expert: para previsão de séries temporais no supply chain. Revista Mundo Logística, Brasil, v.3, n.15, p. 74-82, mar./abr. 2010.

SALGADO JÚNIOR, A. P.; SEGATTO, M.; CALIA, R. C.; FIOROTTO, J. A.; BERTON, L. T. A tecnologia da informação como suporte ao ajuste da previsão da demanda: um estudo de caso em uma empresa de bebidas carbonatadas. Revista Produção Online, Brasil, v. 10, n. 3, p. 621-648, 2010.

SANTOS, A. A. P.; COSTA JR, N. C. A.; COELHO, L. S. Computational intelligence approaches and linear models in case studies of forecasting Exchange rates. Expert Systems with Applications, Brasil, v. 33, p. 816-823, 2007.

SANTOS FILHO, E, L.; SILVA, W. V.; VEIGA, C. P.; TORTATO, U. Previsão dos retornos do Ibovespa utilizando redes neurais artificiais feedforwards evolutivas. Revista Produção Online, Brasil, v. 11, n. 4, p. 1114-1140, 2011.

SONG, H.; LI, G. Tourism demand modeling and forecasting - a review of recent research. Tourism Management, Hong Kong, v. 29, p. 203-220, 2008.

SOUTO, D. P.; BALDEÓN, R. A.; RUSSO, S. L. Estudo dos modelos exponenciais na previsão. Sistemas e Informática, Brasil, v. 9, n. 1, p. 97-103, 2006.

SOUZA, G. P.; SAMOHYL, R. W.; MIRANDA, R. G. Métodos simplificados de previsão empresarial. Rio de Janeiro: Editora Ciência Moderna, 2008, 180p. 
SWANSON, N. R.; WHITE, H. Forecasting economic time series using flexible versus fixed specification and linear versus nonlinear econometric models. International Journal of Forecasting, Estados Unidos, v. 13, p. 439-461, 1997.

TAYLOR, J. W. Forecasting daily supermarket sales using exponentially weighted quantile regression. European Journal of Operational Research, Reino Unido, v. 178, n. 1, p. 154-167, 2007.

TERASVIRTA, T.; DIJK, D. van; MEDEIROS, M. C. Linear models, smooth transition autoregressions, and neural networks for forecasting macroeconomic time series: a re-examination. International Journal of Forecasting, Suécia, v. 21, p. 755-774, 2005.

TERUI, N.; DIJK, H. K. van. Combined forecasts from linear and nonlinear time series models. International Journal of Forecasting, Japão, v. 18, p. 421-438, 2002.

TURRADO GARCÍA, F.; VILLALBA, L. J. G.; PORTELA, J. Intelligent system for time series classification using support vector machines applied to supply- chain. Expert Systems with Applications, 2012. http://dx.doi.org/10.1016/j.eswa.2012.02.137.

VEIGA, C. P. Análise de metodos quantitativos de previsão de demanda: estudo compativo e desempenho financeiro. 2009. 152 f. Dissertação (Mestrado em Engenharia de Produção e Sistemas) - Pontifícia Universidade Católica do Paraná, Brasil, 2009.

VEIGA, C. R. P.; VEIGA, C. P.; DUCLÓS, L. C. The Accuracy of demand forecasting models as crtitical problem to financial performance in the food industry. Future Studies Research Journal: Trends and Strategies, Brasil, v. 2, n. 2, p. 81-104, 2010.

XIE, J.; LEE, T. S.; ZHAO, X. Impact of forecasting error on the performance of capacitated multi-item production systems. Computers \& Industrial Engineering, China, v. 46. p. 205-219, 2004.

YOKUM, J. T.; ARMSTRONG, J. S. Beyond accuracy: comparison of criteria used to select forecasting methods. International Journal of Forecasting, Estados Unidos, v. 11, p. 591-597, 1995.

WANG, J.; LI,L.; NIU, D.; TAN, Z. An annual load forecasting model based on support vector regression with differential evolution algorithm. Applied Energy. China. v. 94, p. 67-70, 2012.

ZENG, A. Z. Eficiency of using fill-rate criterion to determine safety stock: a theoretical perspective and a case study. Production and Inventory Management Journal, Estados Unidos, v. 41, n. 2, p. 41-44, 2000. 
ZHANG, G. P. Time series forecasting using a hybrid ARIMA and neural network model. Neurocomputing, Estados Unidos, v. 50, p. 159-175, 2003.

ZOTTERI, G.; KALCHSCHMIDT, M.; CANIATO, F. The impact of aggregation level on forecasting performance. International Journal of Production Economics, Itália, v. 93-94, n. 8, p. 479-491, 2005.

ZOU, H. F.; XIA, G. P.; YANG, F. T.; WANG, H. Y. An investigation and comparison of artificial neural network and time series models for Chinese food grain price forecasting. Neurocomputing, China, v. 70, p. 2913-2923, 2007.

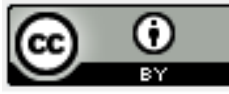

Artigo recebido em 15/03/2011 e aceito para publicação em 07/08/2012. 